\title{
Bases psicosociales del prejuicio étnico en el Perú: el caso de las categorías afroperuanos, peruanos andinos y peruanos blancos
}

\section{Psychosocial bases of ethnic prejudice in Peru: the case of Afro-Peruvian, Andean-Peruvian and White Peruvians categories}

\author{
Henry Rául Guillén Zambrano, Rosa María Luisa Martina Cueto Saldívar, Giacomo Olano Raffo \\ Grupo de Psicología Política y Social - Pontificia Universidad Católica del Perú
}

(Rec: abril de 2018 - Acept: noviembre de 2018)

\begin{abstract}
Resumen
Tomando como base una muestra de personas de clase media de la ciudad de Lima $(n=194)$ se analiza la relación entre variables ideológicas, emocionales y de contenido estereotípico con la valoración personal y percibida de tres categorías étnico raciales: peruanos andinos, peruanos blancos y afroperuanos. Los resultados permiten visibilizar el papel de las variables ideológicas en los niveles de prejuicio dirigidos a dichas categorías, principalmente a las categorías blanco y afroperuano. Además, se hallaron impactos específicos de dimensiones estereotípicas tradicionalmente asociadas con la categoría blanco, andino y afroperuano, como la competencia, lo moral y lo positivo en términos de calidez, respectivamente. Así también, se identifican impactos específicos de las emociones asociadas a cada categoría, principalmente a la categoría peruano blanco y peruano andino. Se analizan además las diferencias obtenidas en los niveles de conservadurismo por sexo. Finalmente, se discute lo hallado a la luz de la evidencia sistemática hallada en otras investigaciones en contextos nacionales.
\end{abstract}

Palabras clave: Prejuicio étnico-racial, ideología política, estereotipos, emociones.

\begin{abstract}
Based on a sample of middle-class people from the city of Lima $(n=194)$, the relationship between ideological, emotional and stereotypical content variables is analyzed with the personal and perceived value of three ethnic-racial categories: Andean Peruvians, White Peruvians, and Afro-Peruvians. The results allow visualizing the role of ideological variables in the levels of prejudice directed to these categories, mainly to the White and Afro-Peruvian categories. Moreover, specific impacts of stereotypical dimensions traditionally associated with White, Andean and Afro-Peruvian categories were found, such as competition, moral and the positive in terms of warmth, respectively. Additionally, specific impacts of the emotions associated with each category are identified, mainly to the White and Andean-Peruvian. Furthermore, the differences obtained in the levels of conservatism by sex are also analyzed. Finally, findings are discussed in the light of the systematic evidence found in other investigations in national contexts.
\end{abstract}

Keywords: Ethnic-racial prejudice, political ideology, stereotypes, emotions. 
El análisis histórico de las relaciones entre los colectivos que estructuran la sociedad peruana revela inequidades y jerarquías, consistentes en una hegemonía de lo blanco sobre lo mestizo y/o lo indígena, generando impactos en términos económicos, sociales e identitarios (Drinot, 2006; Thorp \& Paredes, 2011; Zapata \& Rojas, 2013). La estructura social nacional revela dinámicas de diferenciación, jerarquización y exclusión social (Cueto, 2017), que inclusive han llevado a situaciones de conflicto y violencia colectiva, como el vivido entre 1980 y el 2000 (CVR, 2004; Defensoría del Pueblo, 2014).

La evidencia revela relaciones intergrupales jerárquicas de categorías sociales afianzadas históricamente en el Perú (Drinot, 2006; Espinosa, Calderón-Prada, Burga \& Güímac; 2007). En esa línea, la psicología social plantea que dichas situaciones se sostienen en creencias y valoraciones específicas sobre categorías sociales que configuran el escenario de dinámicas de identificación, representación cognitiva y aproximación afectiva (Espinosa et al., 2007; Pancorbo et al., 2011; Genna \& Espinosa, 2012). Al respecto, en el marco de los estudios sobre identidad nacional en el Perú y Latinoamérica, se identifica un interés por distinguir entre subgrupos basados en diferencias culturales, geográficas y/o étnico-raciales (Espinosa, 2003; Espinosa, 2011; Rottenbacher \& Espinosa, 2010), tomando en cuenta los contenidos estereotípicos y las valoraciones de estas categorías.

Recientemente se viene profundizando en los factores ideológicos que explicarían también estas dinámicas intergrupales (Espinosa, 2011; Espinosa et al., 2007) y de discriminación, dado que ciertas situaciones de contacto intergrupal evidenciarían evaluaciones en términos de amenaza y/o oportunidad. Estas generarían respuestas discriminatorias, sustentadas en estereotipos negativos, valoraciones no favorables e ideologías que promueven la jerarquía y la tradición (Espinosa et al., 2007; Rottenbacher, Espinosa \& Magallanes, 2011).

Conceptualmente, la ideología política es entendida como un sistema de creencias centrales compartidas grupalmente. Dicho sistema está cargado de emociones, creencias y razonamientos sobre la realidad y la forma en cómo debería gobernarse la sociedad (Jost, Federico, \& Napier, 2009), comprendiendo significados abstractos útiles para explicar y justificar la realidad social, económica y/o política (Jost, 2006) que organiza, motiva y da sentido al mundo (Brussino, Rabbia, Imhoff, \& García, 2011). La ideología política promueve y justifica acciones individuales o grupales, orientadas a disminuir diferencias entre lo existente y lo ideal (Lonergan, 1999). Así, actuaría como un heurístico, permitiendo simplificar el universo social, ofreciendo una prescripción sobre cómo debería ser (Brussino et al., 2011), generando herramientas para interpretar la realidad socio-política y orientarse actitudinalmente ante temáticas específicas (Brussino et al., 2011; Jost, 2006).

Actualmente, el estudio de la ideología política establece dos dimensiones. Una de ellas, el autoritarismo de ala derecha (RWA) (Altemeyer, 1981), que expresa valores asociados a la seguridad y orden social, focalizado en el establecimiento y mantenimiento del control, y coherente con una visión del mundo como peligroso y amenazante (Etchezahar \& Imhoff, 2017). El RWA se estructura en tres componentes: respeto y obediencia a la autoridad (sumisión autoritaria), conformidad con las normas y principios tradicionales (convencionalismo) y justificación de la violencia frente a la conducta desviada de la norma social (agresión autoritaria) (Altemeyer, 1981, Jost,
2003). Así, individuos con orientación autoritaria verían las normas sociales como entidades absolutas, legitimadas por autoridades externas y cuya implementación es imprescindible (Radkiewicz, 2016).

Por otro lado, la Orientación a la Dominancia Social (SDO), refiere a una visión darwinista del mundo, que concibe la sociedad como escenario de competencia y supervivencia de los más fuertes. Personas con esta orientación se caracterizarán por valorar mejor el poder, se opondrán a la igualdad y tendrán actitudes favorableas hacia la dominación de grupos poderosos sobre las minorías (Sidanius, Pratto, Stallworth \& Malle, 1994; Etchezahar \& Imhoff, 2017).

Sintetizando, la ideología política, desde una aproximación conservadora, estaría a la base de la diferenciación y jerarquización entre grupos sociales, y del mantenimiento del conflicto intergrupal (Sidanius \& Pratto, 1993). Estas percepciones requieren de procesos psicológicos como la categorización social, que permite diferenciar al propio grupo o categoría (endogrupo) de los grupos ajenos (exogrupos) (Fiske, 1998; Stangor, 2000; Tajfel, 1984). Dichas categorías se configuran a partir de características estereotípicas compartidas intragrupalmente, acompañadas por valoraciones derivadas de la evaluación social de estas características (Luhtanen \& Crocker, 1992; Hogg \& Abrams, 2001). En el marco de las relaciones intergrupales, la categorización social se vincula estrechamente con la formación de estereotipos y valoraciones intergrupales (Fiske, 1998; Hogg \& Abrams, 2001).

Los estereotipos, por su parte, son conjuntos de atributos característicos de grupos sociales específicos o de sus miembros, establecidos mediante las interacciones intergrupales, que permiten organizar la información del entorno social (Stangor, 2000). Además, son avalados por estructuras y normas sociales, políticas y culturales que facilitan su estabilidad en el tiempo y entre grupos (Espinosa \& Cueto, 2014). Uno de los principales modelos del estudio de los estereotipos es el Modelo del Contenido Mixto de los Estereotipos (Stereotype Content Model) desarrollado por Fiske y colaboradores (Fiske, Cuddy, Glick \& Xu, 2002). Dicho modelo establece que el contenido de los estereotipos suele agruparse en dos dimensiones: calidez, asociada a personas tolerantes, buenas y sinceras; y competencia, referida a personas inteligentes y competitivas (Fiske, Cuddy \& Glick, 2003; Fiske et al., 2002). En Latinoamérica, el modelo ha sido ampliado, agregando las dimensiones de moralidad, asociada a características como honestos, respetuosos o solidarios; y nacionalismo ideal, asociado a personas críticas, desarrolladas y patriotas (Espinosa et al., 2016).

Dependiendo de la valoración de los contenidos estereotípicos, las actitudes hacia las categorías sociales variarán. De ser negativa daría origen al prejuicio intergrupal, que se traducirá en pensamientos y conductas discriminatorias (Stangor, 2000), avaladas por estructuras políticas y sociales jerárquicas (Sidanius, Pratto, Stallworth \& Malle, 1994). Así, la inclusión de un sujeto en una categoría específica lo ubicará en un nivel dentro del ordenamiento social (Espinosa, 2011; Espinosa et al., 2007).

Los estereotipos, prejuicios y la discriminación se basan generalmente en tres aspectos: edad, sexo y raza (Stangor, 2000), siendo el factor racial predominante en algunos con- 
textos. Así, el racismo refiere a un tipo de prejuicio, basado en la supuesta superioridad de una raza - o categoría social basada en características étnico-raciales - sobre otras, y que normaliza prácticas y expresiones sociales que pueden institucionalizarse, llevando a naturalizar diferencias y trato desigual (Zárate, 2009).

En Latinoamérica, el racismo se ha caracterizado generalmente como anti-indigenista y anti-negro (Espinosa \& Cueto, 2014). Sin embargo, no existe consenso claro al establecer diferencias étnico-raciales, dado que diversos elementos sociales, económicos y culturales se usan, en conjunto con los rasgos étnicos, para discriminar y promover la desigualdad (Espinosa, 2011). La evidencia refiere que la pirámide social latinoamericana estará marcada por diferencias para acceder a oportunidades y poder entre grupos de mayor o menor estatus, sostenidas en características estereotípicas basadas en rasgos étnico/raciales, culturales y económicos, entre otros (Hopenhayn \& Bello, 2000). Así, grupos de bajo estatus carecerán de medios para acceder al poder y tendrán peores condiciones de vida (Chong \& Ñopo, 2007).

La investigación psicosocial refiere que a grupos de menor estatus les serán atribuidas características asociadas a una débil capacidad para incidir en el desarrollo y la imagen nacional (Cueto, 2017), tal es el caso de la población afro-brasileña en Brasil y la población mapuche en Chile (Espinosa \& Cueto, 2014). En el Perú, la diversidad étnica pareciera tener también una connotación negativa, dados el prejuicio y discriminación a diferentes grupos étnicos, a causa de características negativas asociadas a estos (Genna \& Espinosa, 2012) y que significarían un perjuicio para la imagen nacional (Cueto, 2017; Espinosa, 2011). Así, los peruanos andinos son asociados al atraso y la tristeza, a pesar de ser percibidos como trabajadores, honrados y patriotas (Cueto, 2017). Por otro lado, los afroperuanos son percibidos como atrasados y ociosos, pero alegres (Genna \& Espinosa, 2012). Muchas de estas características suponen valoraciones ambivalentes de los miembros de estas categorías, vinculadas con las características culturales del colectivismo latinoamericano, que valora la agradabilidad (Espinosa et al., 2017); no obstante, al percibírseles como menos competentes, son evaluados como alejados de los espacios de poder (Espinosa \& Cueto, 2014; Pancorbo et al., 2011).

Lo expuesto da cuenta de la dificultad para procesar la diversidad, evidenciada en estudios con muestras no representativas de clases medias (Espinosa, 2011; Rottenbacher \& Espinosa, 2010; Thorp \& Paredes, 2011) y de estratos socioeconómicos medios-bajos y bajos de Lima y otras regiones (cf. Espinosa, 2003; Pancorbo et al., 2011). Por otra parte, la dinámica entre prejuicio y discriminación podría generar un círculo vicioso, donde los conflictos intergrupales étnicos generarían y reforzarían emociones de envidia, resentimiento y odio entre grupos de bajo y alto estatus (Espinosa, \& Cueto, 2014). Asimismo, se evidencian emociones de pena y vergüenza ante grupos menos favorecidos, potencialmente derivadas de la conciencia de la exclusión, y que se sostendrían más bien en la vulnerabilidad percibida del grupo minoritario (Cueto, 2017). Por otro lado, la evidencia refiere asociaciones entre altos niveles de SDO y puntajes altos en discriminación y prejuicio hacia grupos considerados de bajo estatus en muestras peruanas e internacionales (Espinosa, 2011; Monsegur et al., 2014; Pratto et al., 1994), así como una tendencia hostil contra estos (Marasca \& Imhoff, 2013; Rottenbacher et al., 2011) por estereotipos de baja competencia, lo que los alejaría del poder (Cueto, 2017; Espinosa \& Cueto, 2014; Espinosa et al., 2007). Así también, se reporta que puntajes bajos en SDO se asocian con posturas más críticas ante situaciones de inequidad (cf. Cueto, Fourment, Seminario \& Fernández, 2014; Rottenbacher \& Schmitz, 2012) y con mayor empatía con colectivos excluidos (Espinosa, 2011). Sobre el RWA, se le ha señalado como un factor predictor de diferentes medidas de prejuicio étnico, en entornos evaluados como ambiguos y proclives a generar respuestas autoritarias (Rottenbacher et al., 2011).

Tomando en cuenta lo expuesto, es pertinente estudiar las relaciones entre la ideología política conservadora y los procesos a la base de las dinámicas intergrupales - estereotipos, prejuicio y emociones - para el caso de tres categorías étnico-raciales en el Perú: peruanos blancos, tradicionalmente vinculados al poder; peruanos andinos, tradicionalmente vinculados al atraso y pobreza; y afroperuanos, tradicionalmente poco representados en estudios similares (Drinot, 2006). Así, el estudio propone como objetivos: (1) dar cuenta de la magnitud de las variables antes señaladas en la muestra, (2) analizar las relaciones entre las mismas para cada una de las categorías étnico-raciales y (3) finalmente, describir el impacto diferenciado de las variables de interés en la valoración personal percibida de cada una de estas categorías.

\section{Método}

\section{Participantes}

En total participaron 194 personas, con edades entre 18 y 59 años y una media de 26 (DE $=9.201$ ). En cuanto a sexo, fueron 99 mujeres (51\%) y 95 hombres (49\%). La mayoría contaba con estudios universitarios o técnicos incompletos (112), seguido de superiores completos (61). La mayoría se encontraba en NSE medio (116), medio alto (48), medio bajo (23) y alto (9). Se tomó en cuenta un muestreo no-probabilístico de tipo incidental (Otzen \& Manterola, 2017), tomando en cuenta la disponibilidad de acceso y la conformación de una muestra congruente con estudios similares (Espinosa, 2008; Genna \& Espinosa, 2012). El reclutamiento fue por redes sociales virtuales, donde cada participante recibió un consentimiento informado que explicitó la naturaleza del estudio, la importancia de la participación y sus derechos.

\section{Instrumentos de medición}

A continuación, se describen cada uno de los seis instrumentos utilizados para la presente investigación que evalúan variables asociadas al prejuicio, las emociones intergrupales, la estereotipia y la ideología política.

Ficha sociodemográfica: se registró el sexo, la edad, el lugar de origen y de residencia, nivel educativo, grupo étnico percibido, nivel socioeconómico percibido, orientación política y ocupación actual.

Escala de Valoración de Grupos Regionales: se usó la adaptación de la escala de Espinosa (2011), usada por Cueto (2017). Se evalúa con una escala Likert donde $1=$ "muy mala" y 5 = "Muy buena", en base a la valoración personal de tres categorías étnico-raciales: "peruanos blancos", "peruanos andinos" y "peruanos afroperuanos". La escala se usó en estudios donde muestra correlaciones directas con indicadores de autoestima y autoestereotipia, dando validez de criterio recurrente y de constructo convergente (cf. Espinosa, 2008; Genna \& Espinosa, 2012). 
Escala Diferencial de Emociones (Izard, 1991): se usó la versión traducida por Espinosa et al. (2007) para evaluar la intensidad de la vivencia de 10 emociones básicas (alegría, sorpresa, tristeza, interés, ira, asco, desprecio, miedo, culpa y vergüenza), ante las diferentes categorías nombradas, mediante una escala tipo Likert donde 1 = "Nada" y 5 = "Mucho". Se reemplazó el ítem "Asco" por "Disgusto" y se agregaron dos emociones (Envidia y Pena). Se obtuvieron buenas confiabilidades: $\alpha$ : .89 para las emociones positivas y $\alpha: .94$ para emociones negativas hacia los peruanos blancos; $\alpha$ : .84 en las emociones positivas y a: .94 para las negativas en los peruanos andinos y en los afroperuanos $\alpha$ : .83 para las emociones positivas y a: .95 para las negativas.

Escala de Estereotipos Colectivos: utilizada por Cueto (2017), basada en la de Espinosa y colaboradores (2016). La versión consta de 22 parejas de adjetivos y sus antónimos agrupados en un ítem ("Honestos-deshonestos"), donde una mayor puntuación corresponde al adjetivo positivo. Se organiza de acuerdo a un diferencial semántico de 7 puntos; donde debe marcarse el espacio que más se asemeje al adjetivo considerado característico de la categoría social.

Los ítems se distribuyen en 3 dimensiones: morales, competentes y negativos. Para cada grupo étnico se obtuvo buenos niveles de confiabilidad en todas las dimensiones: Moralidad $=\alpha: .86$, Competencia $=\alpha: .84$ y Positivo $=\alpha: .79$ para los Blancos; Moralidad $=\alpha: .87$, Competencia $=\alpha: .86$ y Positivo $\alpha: .77$ correspondiente a los Andinos; y Moralidad = $\alpha: .89$, Competencia $=\alpha: .87$ y Positivo $=\alpha: .82$ correspondiente a los Afroperuanos.

Escala de Autoritarismo de Ala Derecha: versión traducida por Rottenbacher y Schmitz (2012) de la Escala de RWA de Zakrisson (2005) de 15 ítems con una escala Likert donde 1 $=$ "Totalmente en desacuerdo" y $5=$ "Totalmente de acuerdo". Contiene ítems como: "Nuestra sociedad estaría mejor si mostráramos tolerancia y entendimiento hacia los valores y opiniones no-tradicionales". La escala ha sido usada en investigaciones con muestras latinoamericanas, ha correlacionado significativamente con la necesidad de cierre cognitivo (Rottenbacher, 2012), justificación de la inequidad (Rottenbacher \& Molina, 2013) y la criminalización de la protesta social (Rottenbacher \& Schmitz, 2013). En esta investigación la confiabilidad fue de $\alpha: .61$.

Escala de Orientación de la Dominación Social: versión traducida por Silván-Ferrero (Silván-Ferrero \& Bustillos, 2007) de la Escala de Sidanius y Pratto (1999) de 16 ítems. Un ejemplo: "El valor que tienen algunos grupos de personas es mayor que el de otros". Cuenta con una escala Likert donde $1=$ "Totalmente en desacuerdo" y 5 = "Totalmente de acuerdo". La escala ha sido utilizada con muestras latinoamericanas, teniendo correlaciones positivas con: autoritarismo de ala derecha (Rottenbacher \& Molina, 2013), actitudes contra la migración (Sidanius et al., 2000) y prejuicios hacia exogrupos de bajo estatus (Pratto et al., 1994). En esta investigación se obtuvo una confiabilidad de a:.63

\section{Procedimiento y análisis de información}

Se diseñó una encuesta virtual que preguntó sobre el deseo de participar, aceptando, inicialmente, un consentimiento informado. Luego se descargaron las respuestas y se trasladaron a una base de datos del paquete estadístico SPSS 22 donde se realizaron: a) Análisis descriptivos y comparaciones inferenciales, básicamente tomando las diferencias por sexo en las distintas variables de estudio, siendo necesario en algunos casos el uso de estadísticos no paramétricos como la Prueba de Mann-Whitney para muestras independientes; b) Análisis de correlación, para identificar las potenciales asociaciones entre variables de estudio tomando en cuenta las categorías étnico-raciales y c) Regresiones múltiples, a nivel de cada categoría, con el fin de analizar el efecto de las variables psicosociales de base sobre los niveles de valoración en cada categoría social.

\section{Resultados}

La Tabla 1 plantea los estadísticos descriptivos centrados en la muestra total y por sexo. A nivel general, se identifican puntajes superiores a la media escalar en casi todas las variables, salvo en el SDO y las emociones negativas hacia los tres grupos.

No se identificaron mayores diferencias por sexo, salvo en los puntajes en SDO y las tres dimensiones estereotípicas de la categoría afroperuano. La prueba de Mann-Whitney reportó que los hombres obtuvieron puntajes en SDO significativamente mayores ( $M d n=2,187$ ) que las mujeres $(M d n=2,000)$, $\mathrm{U}=5,579, \mathrm{p}=0.025$. Sobre la variable Afroperuano Moral, la prueba de Mann-Whitney halló que los hombres tuvieron puntajes significativamente más bajos ( $M d n=4,000)$ que las mujeres ( $M d n=4,222), U=3,919, p=0.044$. La misma tendencia en la variable Afroperuano Competente, los hombres tuvieron puntajes significativamente más bajos (Mdn $=4,625$ ) que las mujeres ( $M d n=4,250), U=3,605, p=0.005$; y en la variable Afroperuano Positivo, los hombres tuvieron puntajes significativamente más bajos ( $M d n=4,000)$ que las mujeres ( $M d n=4,333$ ), $U=3,613, p=0.005$. Pese a que el tamaño del efecto para las variables estereotípicas fue bajo, en el SDO fue mediano. 
Tabla 1. Estadísticos descriptivos de las variables de estudio: desagregación por sexo

\begin{tabular}{|c|c|c|c|c|c|c|c|c|c|}
\hline \multirow[t]{2}{*}{ VARIABLES } & \multicolumn{3}{|c|}{ TOTAL } & \multicolumn{3}{|c|}{ MUJERES } & \multicolumn{3}{|c|}{ HOMBRES } \\
\hline & $\mathrm{n}$ & $\mathrm{M}$ & $\mathrm{DE}$ & $\mathrm{n}$ & $\mathrm{M}$ & $\mathrm{DE}$ & $\mathrm{n}$ & $\mathrm{M}$ & $\mathrm{DE}$ \\
\hline RWA & 194 & 2.617 & 0.585 & 99 & 2.669 & 0.602 & 95 & 2.562 & 0.564 \\
\hline SDO & 194 & 2.154 & 0.674 & 99 & 2.056 & 0.660 & 95 & 2.256 & 0.676 \\
\hline Blanco Moral & 194 & 4.137 & 0.946 & 99 & 4.143 & 1.037 & 95 & 4.132 & 0.846 \\
\hline Andino Moral & 194 & 4.519 & 1.045 & 99 & 4.567 & 1.193 & 95 & 4.470 & 0.867 \\
\hline Afroperuano Moral & 194 & 4.258 & 1.007 & 99 & 4.402 & 1.074 & 95 & 4.108 & 0.913 \\
\hline Blanco Competente & 194 & 4.689 & 0.900 & 99 & 4.764 & 0.976 & 95 & 4.611 & 0.811 \\
\hline Andino Competente & 194 & 4.277 & 1.090 & 99 & 4.321 & 1.176 & 95 & 4.232 & 0.996 \\
\hline Afroperuano Competente & 194 & 4.534 & 1.001 & 99 & 4.677 & 1.107 & 95 & 4.384 & 0.857 \\
\hline Blanco Positivo & 194 & 3.900 & 1.015 & 99 & 3.904 & 1.104 & 95 & 3.896 & 0.919 \\
\hline Andino Positivo & 194 & 4.436 & 0.996 & 99 & 4.449 & 1.165 & 95 & 4.423 & 0.789 \\
\hline Afroperuano Positivo & 194 & 4.361 & 0.971 & 99 & 4.519 & 1.072 & 95 & 4.196 & 0.828 \\
\hline Emociones Positivas Blancos & 194 & 2.747 & 1.102 & 99 & 2.710 & 1.061 & 95 & 2.786 & 1.147 \\
\hline Emociones Positivas Andinos & 194 & 2.744 & 1.077 & 99 & 2.734 & 1.082 & 95 & 2.754 & 1.079 \\
\hline Emociones Positivas Afroperuanos & 194 & 2.747 & 1.132 & 99 & 2.818 & 1.089 & 95 & 2.674 & 1.177 \\
\hline Emociones Negativas Blancos & 194 & 2.130 & 0.995 & 99 & 2.061 & 0.956 & 95 & 2.201 & 1.034 \\
\hline Emociones Negativas Andinos & 194 & 1.965 & 0.961 & 99 & 1.929 & 0.952 & 95 & 2.003 & 0.974 \\
\hline Emociones Negativas Afroperuanos & 194 & 1.873 & 0.963 & 99 & 1.779 & 0.880 & 95 & 1.971 & 1.037 \\
\hline Valoracion personal: Blanco & 194 & 3.351 & 0.828 & 99 & 3.313 & 0.778 & 95 & 3.389 & 0.879 \\
\hline Valoracion percibida: Blanco & 194 & 3.598 & 1.059 & 99 & 3.566 & 1.126 & 95 & 3.632 & 0.990 \\
\hline Valoracion personal: Andino & 194 & 3.588 & 0.785 & 99 & 3.606 & 0.767 & 95 & 3.568 & 0.808 \\
\hline Valoracion percibida: Andino & 194 & 2.675 & 1.098 & 99 & 2.667 & 1.097 & 95 & 2.684 & 1.104 \\
\hline Valoracion personal: Afroperuano & 194 & 3.454 & 0.802 & 99 & 3.455 & 0.836 & 95 & 3.453 & 0.769 \\
\hline Valoracion percibida: Afroperuano & 194 & 2.644 & 1.074 & 99 & 2.646 & 1.119 & 95 & 2.642 & 1.031 \\
\hline
\end{tabular}

Se realizaron análisis de correlación para las variables asociadas a la valoración personal de las/os participantes con respecto a las tres categorías étnico-raciales. Dichas correlaciones se muestran a continuación.

La Tabla 2 presenta las correlaciones referidas a la categoría "Blanco". Se reportan asociaciones medias entre la valoración personal hacia la categoría y la valoración percibida hacia lo blanco, además de las tres dimensiones estereotípicas. Además, se encuentran asociaciones entre la valoración percibida hacia la categoría y las dimensiones moral y competencia. También, el RWA se asoció de manera media-baja con las emociones positivas y negativas hacia la categoría. Las emociones positivas se asociaron positivamente con dos dimensiones estereotípicas: moral y competente. 
Tabla 2. Correlaciones entre las variables de estudio para la categoría étnico-racial "Blanco"

\begin{tabular}{|c|c|c|c|c|c|c|c|c|c|c|}
\hline Variables & 1 & 2 & 3 & 4 & 5 & 6 & 7 & 8 & 9 & 10 \\
\hline Valoracion personal: Blanco & 1 & $315^{* *}$ & .013 & -.130 & -.034 &, $457^{* *}$ & ,359** &, $366 * *$ & .128 &,$- 170 *$ \\
\hline Valoracion percibida: Blanco & & 1 & -.076 & -.105 &,$- 160 *$ &, $246 * *$ & ,280** &, $143^{*}$ & -.009 & -.138 \\
\hline Orientación política de derecha & & & 1 &, $179 *$ &, $210 * *$ & .081 & .002 & .128 & .009 & -.013 \\
\hline RWA & & & & 1 &, $303^{* *}$ & .025 & .133 & .108 &, $248^{* *}$ & ,208** \\
\hline SDO & & & & & 1 &, $145^{*}$ & .001 & ,181* & .127 & .125 \\
\hline Blanco Moral & & & & & & 1 &, $582 * *$ & ,833** &, $267^{* *}$ & -.040 \\
\hline Blanco Competente & & & & & & & 1 &, $523^{* *}$ &, $252^{* *}$ & -.045 \\
\hline Blanco Positivo & & & & & & & & 1 &, $184^{*}$ & -.045 \\
\hline Emociones Positivas Blancos & & & & & & & & & 1 &, $590 * *$ \\
\hline Emociones Negativas Blancos & & & & & & & & & & 1 \\
\hline
\end{tabular}

**. La correlación es significativa en el nivel 0,01 (bilateral).

*. La correlación es significativa en el nivel 0,05 (bilateral).

La Tabla 3 propone las correlaciones referidas a la categoría "Andino". Se identificaron asociaciones medianas y bajas entre la valoración personal de la categoría y la valoración percibida, dimensiones estereotípicas asociadas a lo moral y lo positivo con emociones positivas asociadas a lo andino. Se encontraron asociaciones entre la valoración percibida y las tres dimensiones estereotípicas, junto con emociones positivas asociadas con lo "Andino". El RWA se asoció de manera mediana y baja con las dimensiones moral y positivo de la categoría. La SDO se asoció de manera mediana con las emociones negativas asociadas a lo andino. Las emociones positivas hacia lo andino correlacionaron de manera mediana y baja con las tres dimensiones estereotípicas y las dos formas de valoración: personal y percibida.

Tabla 3. Correlaciones entre las variables de estudio para la categoría étnico-racial "Andino"

\begin{tabular}{|c|c|c|c|c|c|c|c|c|c|c|}
\hline Variables & 1 & 2 & 3 & 4 & 5 & 6 & 7 & 8 & 9 & 10 \\
\hline Valoracion personal: Andino & 1 & ,295** & -.061 & .011 & -.097 & ,349** & ,166* & ,342** & $234 * *$ & -0.07 \\
\hline Valoracion percibida: Andino & & 1 & .028 & ,181* & 0.14 & ,229** & ,223** & ,298** &, $204^{* *}$ &, $181^{*}$ \\
\hline Orientación política de derecha & & & 1 &, $179 *$ &, $210 * *$ & -.062 & -.017 & -.031 & -.076 & .025 \\
\hline RWA & & & & 1 & ,303** & ,203** &, $160 *$ &, $204^{* *}$ &, $196 * *$ &, $164^{*}$ \\
\hline SDO & & & & & 1 & -0.04 & .035 & -0 & -.015 &, $283^{* *}$ \\
\hline Andino Moral & & & & & & 1 & ,700** &, $860 * *$ &, $336 * *$ & -.056 \\
\hline Andino Competente & & & & & & & 1 &, $721^{* *}$ &, $294^{* *}$ & -.106 \\
\hline Andino Positivo & & & & & & & & 1 &, $354^{* *}$ & -.039 \\
\hline Emociones Positivas Andino & & & & & & & & & 1 & $431^{* *}$ \\
\hline Emociones Negativas Andino & & & & & & & & & & 1 \\
\hline
\end{tabular}

**. La correlación es significativa en el nivel 0,01 (bilateral).

*. La correlación es significativa en el nivel 0,05 (bilateral).

La Tabla 4 propone las correlaciones vinculadas con la categoría "Afroperuano". Se reportan asociaciones bajas y medias entre la valoración personal y las tres dimensiones estereotípicas. Además, la valoración percibida se asoció con el RWA y las tres dimensiones estereotípicas. La SDO se asoció de ma- nera baja con las emociones negativas referidas a la categoría. Las emociones positivas se asociaron de manera baja y media con las tres dimensiones estereotípicas de dicha categoría. 
Tabla 4. Correlaciones entre las variables de estudio para la categoría étnico-racial "Afroperuano"

\begin{tabular}{|c|c|c|c|c|c|c|c|c|c|c|}
\hline Variables & 1 & 2 & 3 & 4 & 5 & 6 & 7 & 8 & 9 & 10 \\
\hline Valoracion personal: Afroperuano & 1 & ,188** & .069 & -.029 & -.136 &, $287^{* *}$ &, $259 * *$ &, $310 * *$ & .091 & -0.07 \\
\hline Valoracion percibida: Afroperuano & & 1 & .085 &, $216 * *$ &, $196 * *$ &, $276^{* *}$ &, $251^{* *}$ & $290 * *$ &, $177^{*}$ & ,149* \\
\hline Orientación política de derecha & & & 1 &, $179 *$ &, $210^{* *}$ & .032 & -.030 & .003 & -.077 & .045 \\
\hline RWA & & & & 1 &, $303^{* *}$ & .121 & .083 & .120 & 0.11 &, $171^{*}$ \\
\hline SDO & & & & & 1 & 0 & -.064 & -0.09 & -.015 &, $265^{* *}$ \\
\hline Afroperuano Moral & & & & & & 1 &, $811^{* *}$ &, $907^{* *}$ &, $330 * *$ & -.084 \\
\hline Afroperuano Competente & & & & & & & 1 &, $796^{* *}$ &, $276^{* *}$ &,$- 174^{*}$ \\
\hline Afroperuano Positivo & & & & & & & & 1 & ,323** & -.088 \\
\hline Emociones Positivas Afroperuano & & & & & & & & & 1 &, $471^{* *}$ \\
\hline Emociones Negativas Afroperuano & & & & & & & & & & 1 \\
\hline
\end{tabular}

**. La correlación es significativa en el nivel 0,01 (bilateral).

*. La correlación es significativa en el nivel 0,05 (bilateral).

Finalmente, se llevaron a cabo análisis de regresión múltiple usando el método por pasos para generar modelos sólidos para predecir las variables dependientes asociadas a las valoraciones de las distintas categorías étnico-raciales. La Tabla 5

Tabla 5. Análisis de Regresión para las valoraciones personales describe cada uno de los modelos finales referidos a las valoraciones personales para cada categoría étnico-racial.

\begin{tabular}{|c|c|c|c|}
\hline & $\begin{array}{c}\text { Valoración Personal: Blanco } \\
\text { Modelo Final }\end{array}$ & $\begin{array}{c}\text { Valoración Personal: Andino } \\
\text { Modelo Final }\end{array}$ & $\begin{array}{c}\text { Valoración Personal: } \\
\text { Afroperuano } \\
\text { Modelo Final }\end{array}$ \\
\hline Andino Moral & & $0.262^{* * *}$ & \\
\hline Afroperuano positivo & & & $0.256^{* * *}$ \\
\hline Blanco Moral & $0.348^{* * *}$ & & \\
\hline Emo. Neg. Blancos & $-0.202^{* *}$ & & \\
\hline Emo. Pos. Blancos & $0.150^{*}$ & & \\
\hline RWA & $-0.197^{*}$ & & \\
\hline $\mathrm{F}$ & $17.201^{* * *}$ & $26.592^{* * *}$ & $20.406^{* * *}$ \\
\hline R2 & 0.267 & 0.096 & 0.122 \\
\hline
\end{tabular}

${ }^{*} \mathrm{p}<.05, * * \mathrm{p}<.01, * * * \mathrm{p}<.001$

Sobre la valoración personal hacia la categoría "Blanco", las principales variables predictoras fueron la dimensión estereotípica Blanco Moral, las emociones positivas y negativas asociadas y el RWA. Dicho modelo explicó un $26,7 \%$ de la varianza. Sobre la valoración personal hacia la categoría "Andino", la principal variable predictora fue la dimensión estereotípica Andino Moral. Dicho modelo explicó el 9.6\% de la varianza asociada a dicha categoría. En el modelo referido a la valoración personal hacia la categoría "Afroperuano" se identificó la dimensión estereotípica Afroperuano Positivo como la principal variable predictora, explicando el $12,2 \%$ de varianza.

El mismo procedimiento se realizó para las variables de valoración percibida sobre cada una de las categorías, como se muestra en la Tabla 6 . En el caso de la valoración percibida sobre la categoría "Blanco", las principales variables predictoras fueron la dimensión estereotípica Blanco Competente, la valoración personal y la SDO; el modelo final explicó el $15.5 \%$ de la varianza. Sobre la valoración percibida sobre la categoría "Andino", las principales variables predictoras fueron le dimen- sión Andino Positivo y las emociones negativas; en este caso, el modelo final explicó el $12.6 \%$ de la varianza. Sobre la valoración percibida sobre la categoría "Afroperuano", las principales variables predictoras fueron la dimensión Afroperuano Positivo y la SDO. El modelo final explica el $13.4 \%$ de la varianza. 
Tabla 6. Análisis de Regresión para las valoraciones percibidas

\begin{tabular}{|c|c|c|c|}
\hline & Valoración Percibida: Blanco & Valoración Percibida: Andino & $\begin{array}{c}\text { Valoración Percibida: } \\
\text { Afroperuano }\end{array}$ \\
\hline & Modelo Final & Modelo Final & Modelo Final \\
\hline Andino Positivo & & $0.337^{* * *}$ & \\
\hline Emo. Neg. Andinos & & $0.220 * *$ & \\
\hline Blanco Competente & $0.229 * *$ & & \\
\hline Val. Per. Blanco & $0.308 * *$ & & \\
\hline Afroperuano positivo & & & $0.343^{* * *}$ \\
\hline SDO & $-0.240^{* *}$ & & $0.358 * *$ \\
\hline $\mathrm{F}$ & $11.581^{* * *}$ & $13.790^{* * *}$ & $14.792^{* * *}$ \\
\hline $\mathrm{R} 2$ & 0.155 & 0.126 & 0.134 \\
\hline
\end{tabular}

${ }^{*} \mathrm{p}<.05, * * \mathrm{p}<.01, * * * \mathrm{p}<.001$

\section{Discusión}

El análisis de los estadísticos descriptivos por sexo reveló diferencias significativas con efectos moderados para la variable SDO. Lo obtenido es congruente con investigaciones preliminares que exploraron el fenómeno de invarianza de género (Sidanius, Pratto \& Bobo, 1994; Cárdenas, Meza, Lagues \& Yañez, 2010). Dicho fenómeno plantea que los niveles de SDO de hombres superan al de mujeres y dichos resultados son invariantes transituacional y transculturalmente, lo que reflejaría jerarquías similares en distintos sistemas sociales (Sidanius, Pratto \& Bobo, 1994). A la base de estas diferencias podrían estar, en alguna medida, jerarquías históricas que favorecieron a los hombres en detrimento de las mujeres, brindándoles mayor nivel de poder político y social (Cárdenas et al., 2010). Los resultados son coherentes con lo planteado por Sidanius \& Pratto (1999), en tanto que "sexo/género" conforma una de las tres estructuras del sistema jerárquico.

El Perú continúa siendo un país con estructuras de género jerárquicas que llevan a distribuciones desiguales de oportunidades y recursos, además de dinámicas de violencia sistemática (INEI, 2015). Nótese también que muchos de estos sistemas se sustentan en la interrelación entre instituciones, medios de comunicación, agentes políticos entre otros, que dan pie a imaginarios colectivos transmitidos en forma de estereotipos y prejuicios, que terminan naturalizando la subordinación femenina (Pecho, 2017). Contextos así, estructuran sistemas de creencias patriarcales que son compartidos y reproducidos individual y colectivamente (Salinas \& Lagos, 2014).

Un segundo eje de discusión apunta a lo obtenido a nivel correlacional. Así, los resultados sobre la categoría blanco refirieron relaciones entre las dimensiones de competencia y moral, valoraciones percibidas y emociones positivas sobre dicha categoría. Lo obtenido es congruente con investigaciones nacionales (Espinosa et al., 2007; Genna \& Espinosa, 2012) donde "lo blanco" es vinculado al alto estatus, y al instrumentalismo, agrupando características ligadas al desarrollo, al éxito $y$ al poder, incluidas en la dimensión de competencia. La evidencia también revela que muchas de las características referidas a lo blanco también se vinculan con el individualis- mo que, en contextos como el peruano, terminan siendo un potencial rasgo de valoración ambivalente, dadas las características colectivistas del país que encuentran incongruencias entre el análisis grupal y nacional (Espinosa et al., 2007; Pancorbo et al., 2011). Como plantean Genna \& Espinosa (2012), la valoración de lo blanco se hace mayor cuando se le vincula al patriotismo y a la solidaridad, alejadas del estereotipo usual de esta categoría, y que explicaría los resultados a nivel de la relación entre la valoración categorial y la dimensión moral. La ambivalencia reportada ha sido una constante en estudios locales con muestras similares (Espinosa et al., 2007) en donde características de la dimensión competencia - como exitosos o capaces - conviven con la falta de patriotismo y la corrupción, vinculados a la categoría blanco (Espinosa, 2011; Espinosa et al., 2007). No obstante, evidencia cualitativa refiere que estas características se encuentran a la base de un conjunto de oportunidades económicas y sociales asociadas a la categoría blanco y que facilitan el fortalecer sus capacidades, así como también las distancias en términos de contacto intergrupal y la discriminación (Pancorbo et al., 2011).

Como refieren Pancorbo y colaboradores (2011), las razones de base del estereotipo y prejuicio sobre lo blanco se enmarcan en visiones sobre las desigualdades y la exclusión latentes en el Perú (c.f. Galarza, Kogan \& Yamada, 2011). Cabe resaltar que los estudios nacionales sobre esta categoría difieren en sus resultados valorativos, siendo la población de nivel socioeconómico bajo la que más reporta valoraciones negativas, a diferencia de estudios con niveles socioeconómicos medio y alto (Pancorbo et al., 2011).

Sobre la categoría andino, los resultados revelaron asociaciones entre los dos tipos de valoración y las dimensiones referidas a lo moral y lo positivo, así como con las emociones positivas. También, las variables ideológicas se asociaron a las dos dimensiones referidas y las emociones negativas sobre la categoría.

Sobre las dimensiones salientes, la evidencia preliminar reporta asociaciones de lo andino con características como trabajadores, patriotas y confiables; elementos a la base de dimensiones como lo moral (Espinosa et al., 2007; Pancorbo et al., 2011; Genna \& Espinosa, 2012). No obstante, también se reportan asociaciones de lo andino con características como 
atraso, subdesarrollo o tristeza, dando cuenta de una imagen ambivalente. Aunque también son de los grupos mejor valorados en algunos estudios (Pancorbo et al., 2011), al igual que con la categoría blanco, la valoración difiere en intensidad dependiendo de los participantes, siendo en contextos socioeconómicamente más bajos donde se encuentran mejores valoraciones, así como en aquellos grupos en los que habría un antecedente familiar vinculado con lo andino. En contraposición, estudios con muestras de nivel medio y medio alto, presentan mayores asociaciones con características como tristeza, atraso, conformismo y fracaso; además de valoraciones menos positivas (Espinosa et al., 2007). Sin embargo, un elemento transversal al nivel socio-económico es la asociación de lo andino con características vinculadas a la dimensión moral, como la solidaridad y la lealtad; cualidades asociadas a sociedades colectivistas vinculadas a procesos de adaptación a condiciones económicas y sociales difíciles (Espinosa et al., 2007; Pancorbo et al., 2011).

Así, la evidencia psicosocial guarda relación con lo reportado en estudios desde las ciencias sociales, donde el carácter trabajador de lo andino también ha sido referido, además de la distancia con conductas de riesgo y una mayor participación ciudadana, elementos relacionados a la dimensión moral (Oboler, 1996; Degregori, 2013). Sobre el vínculo con la participación ciudadana, Degregori (2013) alude que en el caso de lo andino y los fenómenos migratorios, estos grupos han luchado por conquistar derechos, generando sindicatos, asociaciones barriales e innumerables instituciones, llevando así a dinámicas de auto-gobierno y organización distanciadas de las clases medias urbanas de la capital. Estas dinámicas migratorias son consideradas espacios de conquista territorial, que van generando identidades transmitidas generacionalmente (Degregori, Blonde \& Lynch, 1986). No obstante, la construcción estereotípica de lo andino, como en otras categorías étnico-raciales poco valoradas, ha señalado el papel negativo de los medios de comunicación, en tanto transmiten imágenes tergiversadas, superficiales, exóticas y violentas de dicha categoría, manteniendo el prejuicio (Alfaro, 2011; Alarcón, 2014). Sobre la categoría afroperuano, se asociaron variables ideológicas, dimensiones estereotípicas y de valoración, junto a la asociación entre la ideología y la afectividad negativa sobre dicha categoría. Lo hallado guarda coherencia con la evidencia previa, en tanto la construcción estereotípica sobre dicha categoría se vincula a atributos de bajo instrumentalismo, pero alta expresividad y calidez (Genna \& Espinosa, 2012). Así, se mantiene la tendencia ambivalente, así como en el caso andino, aunque en este caso se les identifica como el grupo menos valorado y más discriminado por la sociedad peruana (Espinosa et al., 2007; Pancorbo et al., 2011).

La evidencia guarda relación con estudios focalizados en dicha población, que reportan experiencias de discriminación directa e indirecta, detonando identidades con matices ambivalentes, entre la reivindicación y el rechazo identitario (Benavides, Torero \& Valdivia, 2006). En el caso de las mujeres, estudios revelan que las afroperuanas experimentan más racismo y discriminación; siendo estas experiencias materializadas en espacios como el trabajo o la escuela, y en muchos casos, llevando a estereotipos con características hiper-sexualizadas (Centro de Desarrollo de la Mujer Negra Peruana, 2011) o rasgos enquistados como la relación de lo afroperuano con el baile y el deporte, pero no con la competitividad a nivel académico (Sulmont, 2005).
Finalmente, se identificó mediante análisis de regresión el impacto diferenciado de variables ideológicas, estereotípicas y emocionales en la valoración personal y percibida de las distintas categorías étnico raciales. En ese sentido, en el caso de la categoría blanco intervienen variables ideológicas, el RWA en lo personal y el SDO en lo percibido, variables emocionales y variables estereotípicas en los dos tipos de valoraciones evaluadas. Por otro lado, en la valoración personal de lo andino y lo afroperuano, intervienen básicamente variables de tipo estereotípico.

Lo hallado sobre lo andino y lo afroperuano guarda relación con evidencia previa en tanto lo "andino moral" y lo "afroperuano positivo" son características reportadas sistemáticamente como descriptores de estos grupos (Espinosa et al., 2007; Genna \& Espinosa, 2012). Sobre lo blanco, es saliente el papel de las emociones, con impacto de las emociones negativas y positivas, siendo más fuerte el efecto de las primeras. Sobre el papel del "blanco moral", si bien no fue la dimensión mejor evaluada a nivel descriptivo, se adscribe como un factor predictor en la valoración personal de la categoría. Como mencionan Genna \& Espinosa (2012), la valoración hacia lo blanco es mayor cuando la categoría es asociada a características de patriotismo y solidaridad, elementos de base en la dimensión moral estereotípica.

Además, la evidencia corrobora la importancia de variables ideológicas al analizar el prejuicio,. Tal es el caso de la SDO en la valoración percibida hacia los blancos y afroperuanos, siendo negativo el impacto hacia la primera categoría y positivo hacia la segunda. Al respecto, Genna \& Espinosa (2012) refieren que el tipo de valoración hacia categorías sociales se refuerza en la manera en que grupos de alto estatus dirigen sus prejuicios, pasando por nuevas formas de expresión, como el racismo aversivo (Gaertner \& Dovidio, 2004), sostenidos muchas veces por personas que niegan ser racistas, pero que expresan su incomodidad hacia el contacto, así como frialdad y falta de empatía hacia las minorías.

De esta manera, la SDO se estructura como una variable ideológica que facilita sostener jerarquías sociales, subordinación grupal y atribución de rasgos positivos al endogrupo y negativos a los grupos subordinados (Cárdenas et al., 2010). Dichas atribuciones podrían derivar en nuevas formas de sustentar el prejuicio sutil, en la medida que la SDO es una variable de justificación del sistema y permitiría mantener ciertas convenciones vinculadas al ordenamiento social (Jost \& Thompson, 2000).

Desde el lado del RWA, causa curiosidad su impacto negativo en la valoración personal sobre lo blanco, en tanto ha sido normalmente una medida predictora del prejuicio étnico, principalmente hacia categorías de bajo estatus (Rottenbacher et al., 2011). Entre las posibles razones, podríamos encontrar las características de los/as participantes, pues si bien muchos/as provienen de estratos socioeconómicos medio y medio-altos, pertenecen también a entornos educativos que promueven el cuestionamiento de dinámicas autoritarias e históricamente jerárquicas. Esto abre la necesidad de ampliar la exploración a grupos del mismo nivel socioeconómico, pero de entornos más tradicionales a nivel social y relacional.

Otro resultado a profundizar tendría que ver con las variables explicativas de la valoración personal, dado que el modelo 
para los blancos presenta una mayor cantidad de variables independientes y una mayor varianza explicada. Por otra parte, en los modelos para los andinos y afroperuanos se reporta una variable predictora para cada caso. Valdría la pena explorar otras variables que podrían estar a la base del prejuicio étnico-racial, como los valores individuales y/o colectivos, tal como se hizo en otros estudios nacionales (Espinosa et al., 2007). Otra ruta a explorar serían los alcances desde la Teoría del Contacto Intergrupal (Pettigrew \& Tropp, 2006; Pettigrew \& Tropp, 2008) y el rol de la ansiedad integrupal producto de la amenaza percibida, la empatía con el exogrupo y el nivel de conocimiento sobre las categorías evaluadas; todas estas rutas asociadas a la disminución del prejuicio (Pettigrew \& Tropp, 2008).

Finalmente, el estudio permitió corroborar que los elementos a la base del prejuicio étnico-racial son diversos, y agrupan no solo contenidos estereotípicos, sino también variables ideológicas y emocionales focalizadas. Estas últimas con un potencial explicativo mayor en el caso del prejuicio socialmente percibido.

\section{Referencias}

Altemeyer, B. (1981). Right-wing authoritarianism. Winnipeg, Canada: University of Manitoba press.

Brussino, S. A., Rabbia, H. H., Imhoff, D. \& García, A. P. P. (2011). Dimensión operativa de la ideología política en ciudadanos de Córdoba. Psicología Política, (43), 85-106.

Bustamante, R. (1986). Raza e identidad social positiva y negativa en Lima. En F. León (Ed.), Psicología y Realidad Peruana. El aporte objetivo (pp. 107-130). Lima, Perú: Mosca Azul Editores.

Cárdenas, M., Meza, P., Lagues, K. \& Yañez, S. (2010). Adaptación y validación de la Escala de Orientación a la Dominancia Social (SDO) en una muestra chilena. Universitas Psychologica, 9(1), 161 - 168.

Chong, A. \& Ñopo, H. (2007). Discrimination in Latin America: An elephant in the room? Inter-American Development Bank. Working Paper 614. Recuperado de http://www.iadb.org/en/research-and-data/ publication-details,3169.html ?displaytype =\&pub_ id=WP\%2D614

Comisión de Entrega de la CVR (2004). Hatun Willakuy. Versión abreviada del Informe Final de la Comisión de la Verdad y Reconciliación. Lima: Comisión de Entrega de la CVR.

Contreras, C. (2015). La desigualdad de la distribución de ingresos en el Perú: Orígenes históricos y dinámica política y económica. Lima, Perú: Fondo Editorial de la PUCP.

Cueto, R. (2017). Estudios sobre relaciones intergrupales, identidades colectivas e ideología política en dos regiones del Perú (Tesis de doctorado). Pontificia Universidad Católica del Perú, Lima: Perú

Cueto, R. M., Fourment, K., Seminario, E. \& Fernández, A. (2014). Orientación a la dominancia y representaciones sociales de Estado mercado y política en estudiantes universitarios de Lima, Perú. En M. Rodríguez \& G. Grondona (Coord.), Juventud y política: Cambios sociopolíticos en América del Sur. Quito: Editorial Universitaria Abya-Yala.

Defensoría del Pueblo (2014). Estado de los conflictos socia- les. Recuperado de http://www.defensoria.gob. pe/modules/Downloads/documentos/Infografia-Reporte-de-Conflictos-130.pdf

Drinot, P. (2006). Construcción de nación, racismo y desigualdad: Una perspectiva del desarrollo institucional en el Perú. En J. Crabtree (Ed.), Construir instituciones: Democracia, desarrollo y desigualdad en el Perú desde 1980 (pp. 11-31). Lima: Fondo Editorial de la PUCP, Centro de Investigación de la Universidad del Pacífico, IEP.

Espinosa, A. \& Cueto, R.M. (2014). Estereotipos raciales, racismo y discriminación en América Latina. En $\mathrm{E}$. Zubieta, J. Valencia y G. Delfino, Psicología Social y Política: Procesos teóricos y estudios aplicados (pp. 352-361). Buenos Aires, Argentina: EUDEBA.

Espinosa, A. (2011). Estudios sobre identidad nacional en el Perú y sus correlatos psicológicos, sociales y culturales (Tesis Doctoral). Universidad del País Vasco, San Sebastián, España.

Espinosa, A., Acosta, Y., Valencia, J., Vera, A., Soares, A., Romero, J. C. \& Beramendi, M. (2016). Calidez, competencia, moralidad y nacionalismo ideal como dimensiones autoestereotípicas en seis países de Latinoamérica. Avances en Psicología Latinoamericana, 34(2), 395-413.

Espinosa, A., Calderón-Prada, A., Burga, G. \& Güímac, J. (2007). Estereotipos, prejuicios y exclusión social en un país multiétnico: El caso peruano. Revista de Psicología, 25, 295-338.

Espinosa, A., Soares da Silva, A., Contreras, C., Cueto, R.M., García, A., Ortolano, F., Vera, A. (2017). Identidad Nacional y sus relaciones con la ideología y el bienestar en 5 países de América Latina. Avances en Psicología Latinoamericana, 35(2), 351-374.

Etchezahar, E. \& Imhoff, D. (2017). Relaciones entre el autoritarismo y la dominancia social de acuerdo al nivel de contraste ideológico del contexto socio-político argentino. Psicología, Conocimiento y Sociedad, 7(1), 59-75.

Fiske, S. (1998). Stereotyping, prejudice, and discrimination. En D. Gilbert, S. Fiske \& G. Lindzey (Eds.), Handbook of Social Psychology, 2(4), 357-412. Boston, United States: The McGraw-Hill Companies.

Fiske, S. T., Cuddy, A. J. \& Glick, P. (2003). Emotions up and down: Intergroup emotions result from perceived status and competition. En D. M. Mackie, \& E. R. Parrot (Eds.), From Prejudice to Intergroup Emotions: Differentiated Reactions to Social Groups (pp. 247-264). Nueva York, United States: Psychology Press.

Fiske, S. T., Cuddy, A. J., Glick, P., \& Xu, J. (2002). A model of (often mixed) stereotype content: Competence and warmth respectively follow from perceived status and competition. Journal of Personality and Social Psychology, 82, 878-902.

Genna, K. \& Espinosa, A. (2012). Identidad, etnicidad y bienestar social en un contexto socialmente excluyente. Psicologia \& Sociedade, 24(1), 84-93.

Gonzales de Olarte, E. (2005). Crecimiento, desigualdad e ingobernabilidad en el Perú de los 2000. En V. Vich (Ed.), El Estado está de vuelta: Crecimiento, diversidad y democracia (pp. 49-70). Lima, Perú: IEP.

Hogg, M. \& Abrams, D. (2001). Intergroup relations: An overview. En M. Hogg \& D. Abrams (Eds.), Intergroup 
relations: Essential readings (pp.1-17). Philadelphia, United States: Psychology Press.

Hopenhayn, M., \& Bello, A. (2000). Tendencias generales, prioridades y obstáculos en la lucha contra el racismo, la discriminación racial, la xenofobia y las formas conexas de intolerancia. América Latina y el Caribe.[En línea]. CEPAL.

Izard, C. E. (1991). The psychology of emotions. Springer Science \& Business Media.

Jost, J. T. (2006). The end of the end of ideology. American Psychologist, 61(7), 651.

Jost, J. T., Federico, C. M. \& Napier, J. L. (2009). Political ideology: Its structure, functions, and elective affinities. Annual Review of Psychology, 60, 307-337.

Jost, J. T., Glaser, J., Kruglanski, A. W. \& Sulloway, F. J. (2003). Political conservatism as motivated social cognition. Psychological bulletin, 129(3), 339.

Kogan, L. \& Galarza, F. (2014). ¿Discriminas o te discriminan? Un análisis de las percepciones de universitarios de cuatro ciudades del Perú. Lima, Perú: Universidad del Pacífico.

Lonergan, B. (1999). Insight, estudio sobre la comprensión humana. Salamanca, España: Sígueme

Luhtanen, R. \& Crocker, J. (1992). A collective self-esteem scale: Self-evaluation of one's identity. Personality and Social Psychology Bulletin, 18, 302-318.

Marasca, M., Marasca, R. \& Imhoff, D. (2013). Indagación del autoritarismo en la infancia: Vinculaciones con la Orientación de Dominancia Social y lo valores en niños y niñas de la ciudad de Córdoba (Argentina). Interdisciplinaria, 30(1), 139-161.

Monsegur, S., Espinosa, A. \& Beramendi, M. (2014). Identidad nacional y su relación con la dominancia social y la tolerancia a la transgresión en residentes de Buenos Aires. Interdisciplinaria, 31(1), 5 - 23.

Otzen, T. \& Manterola, C. (2017). Técnicas de Muestreo sobre una Población a Estudio. International Journal of Morphology, 35(1).

Pancorbo, G., Espinosa, A. \& Cueto, R. M. (2011). Representaciones estereotípicas y expresión del prejuicio en el Perú: La mirada desde la pobreza. Revista de Psicología, 29, 311-342.

Pratto, J., Sidanius, J., Stallworth, L. \& Mallé, B. (1994). Social Dominance Orientation: A personality variable predicting social and political attitudes. Journal of Personality and Social Psychology, 67, 741-763.

Radkiewicz, P. (2016). Does authoritarianism imply ethnocentric national attitudes: A revised look at the "authoritarian triad" and right-wing ideology. European Journal of Social Psychology, 46(2), 224-236.

Rottenbacher, J. \& Molina, J. (2013). Intolerance to Ambiguity, Political Conservatism, and Justification of Economic, Legal, Educational, and Ethnic Inequality in the City of Lima-Peru. Revista Colombiana de Psicología, 22(2), 253-274.

Rottenbacher, J. (2012). Conservadurismo político, homofobia y prejuicio hacia grupos transgénero en una muestra de estudiantes y egresados universitarios de Lima. Pensamiento Psicológico, 10(1), 23-37.

Rottenbacher, J. M. \& Espinosa, A. (2010). Identidad nacional y memoria histórica colectiva en el Perú. Revista de Psicología, 28(1), 147-174.

Rottenbacher, J. M. \& Molina, J. (2013). Intolerancia a la ambigüedad, conservadurismo político y justificación de la inequidad económica, legal, educativa y étnica en la ciudad de Lima - Perú. Revista Colombiana de Psicología, 22(2), 253-274.

Rottenbacher, J. M. \& Schmitz, M. (2013). Condicionantes ideológicos de la criminalización de la protesta social y el apoyo a la democracia en una muestra limeña. Revista de Psicología de la PUCP, 31(2), 371-394.

Rottenbacher, J. M., Espinosa, A, \& Magallanes, J. M. (2011). Analizando el prejuicio: Bases ideológicas del racismo, el sexismo y la homofobia en una muestra de habitantes de la ciudad de Lima - Perú. Psicología Política, 11(22), 225-246.

Rottenbacher, J., \& Schmitz, M. (2012). Democracia vs neoliberalismo económico. Condicionantes ideológicos de las preferencias políticas y económicas en la ciudad de Lima. Revista mexicana de ciencias políticas y sociales, 57(214), 111-140.

Sidanius, J. \& Pratto, F. (1999). Social dominance: An intergroup theory of social hierarchy and oppression. New York, United States: Cambridge University Press.

Sidanius, J., \& Pratto, F. (1993). The inevitability of oppression and the dynamics of social dominance. Prejudice, politics, and the American dilemma, 173-211.

Sidanius, J., Pratto, F \& Bobo, L. (1994). Social Dominance Orientation and The Political Psychology of Gender: A Case of Invariance?. Journal of Personality and Social Psychology, 67(6), 998 - 1011.

Sidanius, J., Levin, S., Liu, J., \& Pratto, F. (2000). Social dominance orientation, anti-egalitarianism and the political psychology of gender: an extension and cross-cultural replication. European journal of social psychology, 30(1), 41-67.

Sidanius, J., Pratto, F., Stallworth, L. M. \& Malle, B. F. (1994). Social dominance orientation: A personality variable predicting social and political attitudes. Journal of personality and social psychology, 67(4), 741.

Silván-Ferrero, M. D. P., \& Bustillos, A. (2007). Adaptación de la escala de Orientación a la Dominancia Social al castellano: validación de la Dominancia Grupal y la Oposición a la Igualdad como factores subyacentes. Revista de Psicología Social, 22(1), 3-15.

Stangor, C. (2000). Overview. En C. Stangor (Ed.), Stereotypes and prejudice: Keyreadings in social psychology (pp. 1-16). Michigan, United States: Edwards Brothers.

Tajfel, H. (1984). Intergroup relations, social myths and social justice in social psychology. The social dimension, 2, 695-715.

Thorp, R. \& Paredes, M. (2011). La etnicidad y la persistencia de la desigualdad. El caso peruano. Lima: IEP.

Uccelli, F. \& García Llorens, M. (2016). "Solo zapatillas de marca”. Jóvenes limeños y los límites de la inclusión desde el mercado. Lima, Perú: IEP.

Zapata, A. \& Rojas, R. (2013). ¿Desiguales desde siempre? Miradas históricas sobre la desigualdad. Lima, Perú: IEP.

Zavala, V. \& Córdova, G. (2010). Decir y callar. Lenguaje, equidad y poder en la universidad peruana. Lima, Perú: Fondo Editorial PUCP 